Original article

\title{
Changes of oxidation during use the food diet with deuterium depleted water in laboratory animals with purulent inflammation
}

\author{
Oxana Artsybasheva ${ }^{1}$, Ekaterina Barysheva ${ }^{2}$, Denis Shashkov ${ }^{1}$, Roman Vlasov ${ }^{1}$, Elena Tekutskaya ${ }^{1}$ \\ ${ }^{1}$ Kuban State University, Krasnodar, Russia \\ ${ }^{2}$ Kuban State Medical University, Krasnodar, Russia
}

Received 8 April 2014, Revised 19 May 2014, Accepted 10 June 2014

(C) 2014, Artsybasheva O., Barysheva E., Shashkov D., Vlasov R., Tekutskaya E.

(C) 2014, Russian Open Medical Journal

\begin{abstract}
Objective - To identify quantitative changes of the deuterium content, the intensity of free radical oxidation and antioxidant status of the blood system, as well as the effect of water with a modified isotope composition with low deuterium content on indicators of free radical oxidation of tissues in laboratory animals under physiological conditions and in inflammatory processes.

Materials and methods - The object of the study was blood and homogenates of organs (liver, kidney) of male rats. The basis of the model of oxidative stress was a well-known model of wound healing proposed by L.A. Mamedov based on the surgical treatment of abscess model, and we performed its modification in the course of experimental studies. Determination of deuterium concentration in the plasma was carried out using nuclear magnetic resonance. The method of luminol-dependent $\mathrm{H}_{2} \mathrm{O}_{2}$-induced chemiluminescence was used for the detection of relatively unstable chemically active radicals in the plasma. Stable radicals detect by the EPR spectrometry.

Results and Conclusion - Thus, it should be noted that blood plasma demonstrates reliable reduction in deuterium concentration when using deuterium depleted water that continues until the values of 90-100 ppm thereafter remaining practically unchanged. At the same time, deuterium depleted water affects prooxidant-antioxidant system of the body reducing the intensity of free radical oxidation and restoring the capacity of the endogenous antioxidant system.
\end{abstract}

Keywords: antioxidants, oxidative stress, deuterium depleted water, electron paramagnetic resonance

Cite as Artsybasheva O, Barysheva E, Shashkov D, Vlasov R, Tekutskaya E. Changes of oxidation during use the food diet with deuterium depleted water in laboratory animals with purulent inflammation. Russian Open Medical Journal 2014; 3: 0201.

Correspondence to Oxana Artcybasheva. Address: 149, Stavropol'skaya str., Krasnodar, 350040, Russia. Tel.: +79284098789. E-mail: artsybashevao@mail.ru

\section{Introduction}

The existence of the human body in permanent contact with adverse environmental factors is impossible without the proper functioning of its non-specific defense systems. The weakening of non-specific resistance is observed in many diseases and specific physiological conditions, which is characterized by the reduction of adaptive responses and contributes to the development of various disorders of homeostasis leading to adverse outcomes, reducing of mental and physical work capacity. Antioxidant system (AOS) is one of the key systems of nonspecific defense, which maintain the ratio of prooxidant and antioxidant factors in the body at the physiological level $[1,2]$. Despite the fact that prooxidants - free radicals (superoxide anion radical, nitric oxide, hydroxyl radical, alkyls, alkoxyls and peroxides) and reactive molecules (hydrogen peroxide, hypochlorite anion, hydroperoxides and peroxynitrite) are actively involved in the regulation of many intracellular processes [3-7] including immune mechanisms, neutralization of xenobiotics, apoptosis, metabolism of bioactive compounds (prostaglandins, biogenic amines), metabolism of bone tissue and the oxidation of hemoglobin in cases of imbalance in prooxidantantioxidant system of the organism with the prevalence of prooxidant factors. The latter factors start to possess damaging effect at the molecular and cellular level, which is accompanied by a set of typical pathological changes in organs and tissues, called as "oxidative stress" $[8,9]$.

Taking into account the important role of free radical reactions of oxidation (FRRO) in the regulation of physiological processes and the development of pathological conditions an active search of ways for pharmaceutical and non-pharmacological correction of disorders that are developed in conditions of oxidative stress (OS) is being continued in modern biology, prevention and clinical medicine in order to prevent formation or reduce complications in a variety of diseases (diabetes, atherosclerosis, bronchial asthma, oncopathology, rheumatoid arthritis, neurodegenerative and other diseases) where OS is essential in the pathogenesis [10-14]. The possibility of nutritional correction of oxidative metabolism in the body becomes of considerable interest, which is primarily due to the ability of nutrients to have a significant influence on the health, work capacity and life span, so now, in addition to the optimal balance of nutrients and minerals, an estimation of their effect on the endogenous AOS is carried out [15-17]. One of the most promising nutrients for the correction of the antioxidant capacity of the body is water with a modified isotopic composition (WMIC), for example, water with low content of deuterium [18]. 


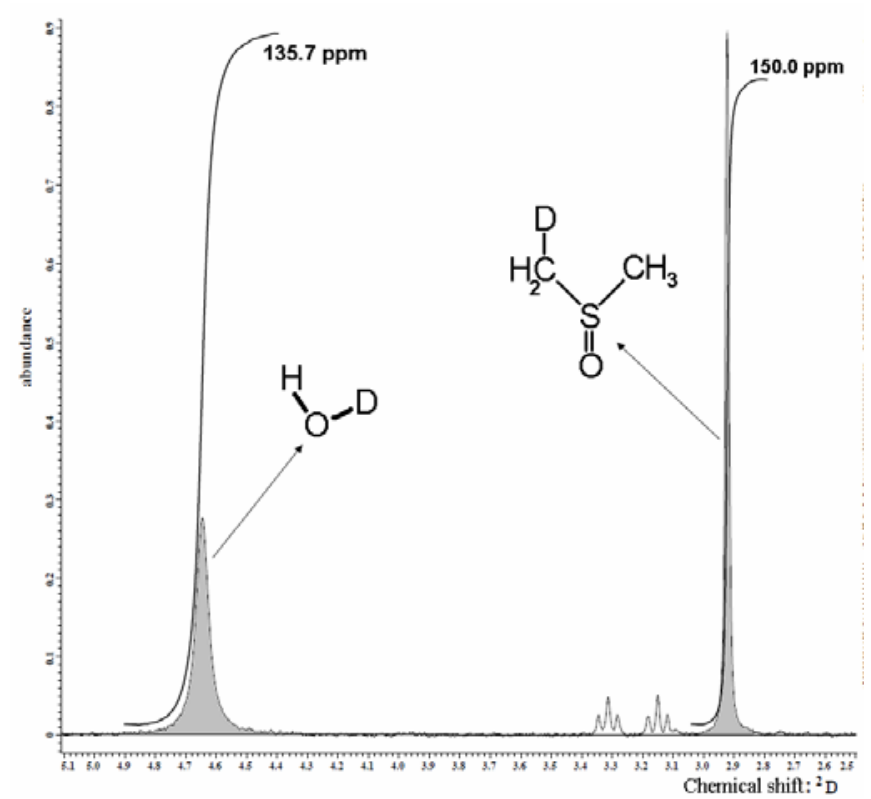

Figure 1. Integral intensity ratio of 2D NMR HDO signal relatively to 2D NMR DMSO-D1 signal

We know that people consume up to 2-3 liters of fluid per day under physiological conditions, so the change in the structure of nutrition caused by WMIC with low deuterium content can affect the values of AOS. Recently all over the world various effects of WMIC with low deuterium content have been actively studied; its main effect on the body is the gradual reduction of deuterium content in biological fluids and tissues due to isotope exchange reactions. The scientific literature often describes biological effects of WMIC, whereas the molecular mechanisms of its action on the body have not been fully examined yet. WMIC with low deuterium content affects the growth of tumor cells of various cultures [1921], possesses immunomodulating property [22-24] and affects the metabolism of hydrogen peroxide in the liver [25]. All above examples show the great role of isotope composition of water for molecular processes in the body, and therefore the addition of WMIC with low deuterium content to people's diet in states accompanied by the development of the OS may increase the potential of endogenous AOS and provide benefit in the prevention of complications [26]. Thus, the increasing use of WMIC with low deuterium content in people with various pathological conditions, for health improvement (fitness), as well as in professional sports requires further study on the mechanisms of its action, which will allow more efficient use of its effects for prophylaxis and at various clinical conditions.

Objective: To identify quantitative changes of the deuterium content, the intensity of free radical oxidation and antioxidant status of the blood system, as well as the effect of water with a modified isotope composition with low deuterium content on indicators of free radical oxidation of tissues in laboratory animals under physiological conditions and in inflammatory processes.

\section{Material and Methods}

The object of the study was blood and homogenates of organs (liver, kidney) of male rats with weight of 90-100 g. The rats were divided into the following groups: group No.1 (which were administered distilled mineralized water (158 ppm) for 30 days, $n=40$ ), group No.2 (which were administered distilled mineralized water
(158 ppm) for 30 days and suffered from purulent inflammation of soft tissue, $n=40$ ), group No.3 (which were administered distilled mineralized water with reduced deuterium content $(40 \mathrm{ppm})$ for 30 days and suffered from purulent inflammation of soft tissue, $n=40$ ).

Water with low deuterium content was obtained at the facility developed at Kuban State University [27, 28]. The initial concentration of deuterium in the produced water was $40 \mathrm{ppm}$.

Two-stage model of oxidative stress was used while modeling purulent wounds in rats. The first stage was a acute stage of oxidative stress, and it was simulated by creating intermuscular abscess in the soft tissues of the long muscles of the back of laboratory animal by using an implanted foreign body. The second stage reflected the chronic stage of oxidative stress, and it was simulated by purulent wound that was formed naturally while draining the abscess and removal of the foreign body.

The basis of the model of oxidative stress was a well-known model of wound healing proposed by L.A. Mamedov based on the surgical treatment of abscess model, and we performed its modification in the course of experimental studies [29].

In order to create a model of abscess, one cut and shaved rat's hair on the middle and lower thirds of the back before the experiment. Then, under local anesthesia with novocaine $0.5 \%$ solution - $10 \mathrm{ml}$ syringe needle was applied causing damage of soft tissue (in the area of the long back muscles) at the depth of $3 \mathrm{~cm}$ and the width of $2 \mathrm{~cm}$ in the intended area of abscess formation. On the day of the experiment a $3 \mathrm{~cm}$ length incision of compromised area was made under chloralose-nembutal anesthesia followed by insertion of sterile gauze sponge of $10 \mathrm{~mm}$ diameter impregnated with $1 \mathrm{ml}$ of liquid containing the pathogenic strain of St. aureus into the soft tissues. Primary sutures of the wound were performed.

After one day animals developed a clinic of wound abscess, and the first (acute) period of oxidative stress simulation started. The sutures were removed in 5 days after infection, corresponding to the transition to the second stage of oxidative stress.

Local treatment of purulent wound under the salve dressings was performed later up to the complete healing by secondary intention.

Determination of deuterium concentration in the plasma was carried out using nuclear magnetic resonance (NMR) at the pulsed NMR spectrometer JEOL JNM-ECA $400 \mathrm{MHz}$. Spectra recording was carried out at the corresponding resonance frequency of deuterium nuclei - 61.4 MHz. Recording parameters was as follows: $6.7 \mathrm{~s}$ (acquisition time), $20 \mathrm{~s}$ (relaxation delay), $5.6 \mathrm{~ms}(\mathrm{x}$ pulse), $0.15 \mathrm{~Hz}$ (resolution). Recording temperature was $-25^{\circ} \mathrm{C}$, with the accuracy of stabilization of $0.2{ }^{\circ} \mathrm{C}$. Measurements were carried out using $5 \mathrm{~mm}$ ampoule with fixed sealed capillary inside containing mixture of deuterated and undeuterated dimethyl sulfoxide (DMSO) according to defined concentration calibrated scale that produce 2D NMR signal at $3.4 \mathrm{ppm}$ (relative to (CD3) 4Si), while 2D NMR HDO signal is in the range of $4.7 \mathrm{ppm}$ (relative to (CD3) 4Si) (Figure 1).

Processing of obtained spectra consisted in determining of the ratio of integral intensities of $2 D$ NMR HDO signal of the test sample relative to $2 \mathrm{D}$ NMR DMSO-D1 signal, the intensity of which was in turn determined under the same conditions relative to standards - water samples with precisely defined deuterium content ( $3.7 \mathrm{ppm}, 51 \mathrm{ppm}, 150 \mathrm{ppm}$ ). The measurements of each sample were carried out repeatedly to decrease experimental error. The accuracy of deuterium content determination in biological samples was $\pm 2 \mathrm{ppm}$. 
Table 1. Indicators of the deuterium content, the intensity of free radical oxidation and state of the antioxidant system in blood and tissues of the rats using water with modified isotope composition in the diet $(\mathrm{M} \pm \mathrm{m})$

\begin{tabular}{lcccc}
\hline Indicator $\backslash$ Group & $\begin{array}{c}\text { Content of deuterium } \\
\text { in the plasma }\end{array}$ & $\begin{array}{c}\text { MFCL of plasma, } \\
\text { arbitrary units }\end{array}$ & AOA of plasma, nA.s & EPR of kidneys, PMC/g \\
\hline Group $1(n=40)$ & $153.3 \pm 0.4$ & $1.993 \pm 0.024$ & $1246.4 \pm 23.7$ & $608.61 \pm 44.01$ \\
Group 2 $(n=40)$ & $157.5 \pm 0.4$ & $3.058 \pm 0.081 *$ & $948.2 \pm 21.8 *$ & $747.87 \pm 55.13 *$ \\
Group $3(n=40)$ & $96.1 \pm 0.5 *^{+}$ & $2.716 \pm 0.126 *^{+}$ & $1137.1 \pm 24.2 *^{+}$ & $720.66 \pm 57.07 *$ \\
\hline
\end{tabular}

$*-P<0.05$ in comparison with the indicators of group No. 1 ;

${ }^{+}-\mathrm{P}<0.05$ in comparison with the indicators of group No. 2 .
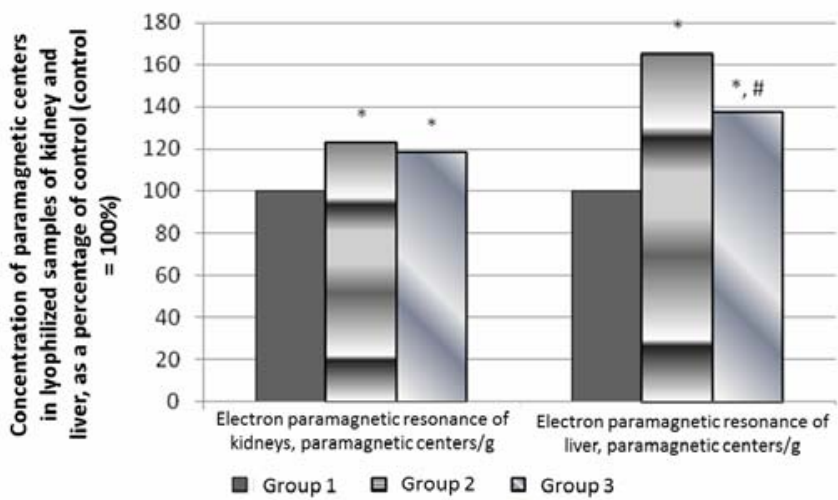

Figure 2. Influence of water with modified composition on values of free radical oxidation in liver and kidneys in laboratory animals with purulent inflammation of soft tissues

$*-P<0.05$ in comparison with the indicators of group 1 ;

$\#-P<0.05$ in comparison with the indicators of group 2;

$\mathrm{PMC} / \mathrm{g}$ - the number of paramagnetic centers in 1 gram of lyophilized tissue.

Measurement of electron paramagnetic resonance (EPR) spectra was performed at room temperature at JES Fa 300 spectrometer (JEOL, Japan) in the X-band. The conditions were as following: microwave power was $1 \mathrm{~mW}$, microwave frequency was $9144 \mathrm{MHz}$ and high-frequency amplitude modulation was $0.1 \mathrm{mT}$. Tissue samples were previously lyophilized (at dehumidification device LS-1000), measured in a quartz ampoule $(5 \mathrm{~mm})$; the mass of the sample in the resonator zone was $0.03 \mathrm{~g}$. The concentration of paramagnetic centers (PMC) in the samples was determined by comparison to the signal of a standard sample (TEMPOL). Integrated intensity of EPR signal in the samples was determined by double numerical rectangular integration method [30].

The EPR spectra of liver samples of the laboratory mice contain anisotropic singlet signal which spin-Hamiltonian parameters correspond to those of stable radicals [31-33]. The EPR spectra of kidney samples are similar.

Given that the EPR method can detect mainly stable radicals [34] the method of luminol-dependent $\mathrm{H}_{2} \mathrm{O}_{2}$-induced chemiluminescence was used for the detection of relatively unstable chemically active radicals in the plasma with hemi lumen tester LT-1 manufactured by Scientific and Production Association Lumin (Rostov-on-Don) in the modification [35-37]. The results obtained in the form of maximum flash chemiluminescence (MFCL) reflected inhibition of FRRO was expressed in arbitrary units (arb. u.) in relation to the flash in control samples without the biological material.

Determination of antioxidant activity (AOA) of blood plasma was carried out additionally to the assessment of the endogenous antioxidant system by amperometric method at antioxidant activity analyzer "Yauza-01-AAA" produced by Scientific and Production Association "Khimavtomatika" OAO (Moscow, Russia) by the method [38]. The method is based on the measurement of electric current, which occurs during the oxidation of biological sample at the surface of the working electrode at the specific potential and comparing the received signal with a standard signal measured under the same conditions. The results were expressed in nanoampere per second ( $\left.\mathrm{nA}^{*} \mathrm{~s}\right)$.

Statistical analysis of the data obtained was carried out by the methods of variation statistics by using Student t-test. Difference was considered as significant at $p<0.05$. Data in the table are presented as the arithmetic mean (M) and the error of the arithmetic mean $( \pm m)$.

\section{Results}

The study found that lowest deuterium content in biological fluids and tissues was typical for animals of group 3, which were administered WMIC with low deuterium content in their diet. Deuterium content was lower by $37.3 \%$ and $38.9 \%$ in comparison to the indicators in group 1 and 2 respectively.

This indicates a reliable $(p<0.05)$ change of the deuterium content in the blood in 30 days after the start of the study (Table 1). At the same time it should be noted that deuterium plasma content ceased to decrease after reaching a value of 90 $100 \mathrm{ppm}$, and its further decrease didn't happen despite the lower levels of deuterium in WMIC ( $40 \mathrm{ppm}$ ) administered to laboratory animals. This suggests the presence of specific bodily mechanisms regulating an isotope composition of biological fluids in physiological range preventing sudden changes in the quantitative content of hydrogen isotopes in different tissues and organs.

\section{Discussion}

When comparing the intensity of free radical formation in lyophilized organs it was found that the liver and kidneys of rats with simulated oxidative stress (Groups 2 and 3 ) demonstrated a significant increase in the concentration of PMC (Figure 2). This indicates a steady prevalence of prooxidant factors on the AOS component at the cellular level. At that, there were more significant changes in liver homogenates from the animals of group 2 where concentration of PMC exceeded the value of a control group 1 by $64.9 \%(P<0.05)$.

This fact indicates an active participation of the liver in the elimination of toxic substances accumulated due to the chronic inflammatory processes resulting in increasing synthesis of active oxygen forms in hepatocytes and the development of OS at the tissue and organ levels. It should be noted that the PMC content in liver homogenates of the $3^{\text {rd }}$ group of animals was also significantly higher than in group $1-$ by $37.1 \%(P<0.05)$, but it was significantly lower than in group $2-$ by $16.9 \%(P<0.05)$, which 
reflects a less pronounced rate of FRRO in hepatocytes of these animals and probably indicates more active tissue components of endogenous AOS or less toxic load on the liver cells from the focus of inflammation [39-42]. This can be explained by the fact that WMIC with low deuterium content activates other non-specific and protective systems, for example possess immunomodulatory effect accelerating the localization mechanisms of pathological agents by means of cellular immunity.

When studying the processes of FRRO in kidney homogenates less pronounced changes were obtained in rats with simulated OS though they were significantly increased in group 2 (by 22.9\%, $\mathrm{P}<0.05$ ) and in group 3 (by $18.4 \%, \mathrm{P}<0.05$ ) in comparison with values of the control group 1 [43-45]. There were not any significant differences in the both experimental groups 2 and 3, which may be caused due to lower specific influence of WMIC with low deuterium content on the endogenous AOS of kidneys or less capacity of low and medium weight hydrophilic toxic substances to activate the free radical processes in the kidney tissue. It is known that some of them (e.g. urea, oligopeptides, and uric acid) may exhibit antioxidant effect by participating in the neutralization of free radicals that reduces the amount of free radicals in the organs of the excretory system $[46,47]$.

Changes in the blood were more significant in nature due to the integrating function of the blood as a biological fluid reflecting the whole spectrum of changes in the body. A significant decrease of $A O A$ in rats in group 2 (by $23.9 \%, P<0.05$ ) was revealed during the study of plasma, while rates in group 3 had a much smaller decrease in $A O A$, and its values significantly exceeded those of the group 3 (by $19.9 \%, P<0.05$ ). Similar changes characterized the reduction of endogenous AOS capacity especially its low molecular component throughout the body. This can lead to various repeated pathological processes and complications. In turn, the blood level of FRRO in rats from group 2 and group 3 was significantly increased $(\mathrm{P}<0.05)$ compared to controls by $53.7 \%$ and $36.7 \%$ respectively (Figure 3 ).

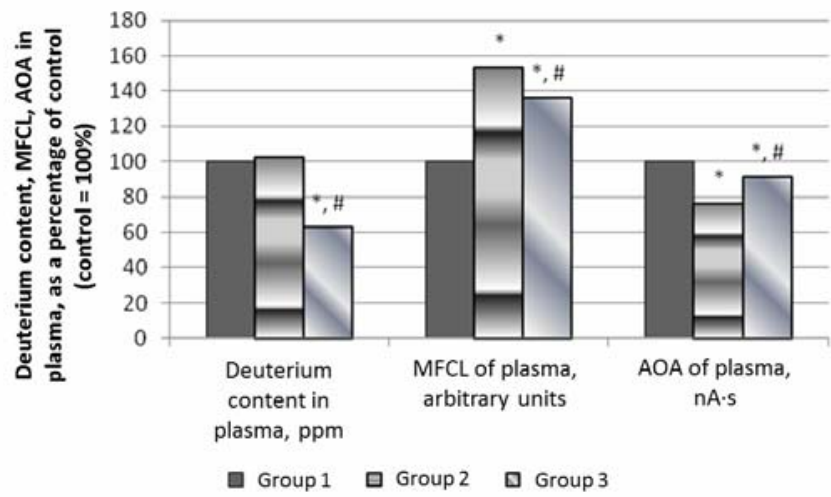

Figure 3. Influence of water with modified composition on the values of deuterium content, MFCH and AOA of blood plasma in laboratory animals with purulent inflammation of soft tissues

* $-\mathrm{P}<0.05$ in comparison with the indicators of group $1 ;$

$\#-P<0.05$ in comparison with the indicators of group 2;

MFCL - maximal flash chemiluminescence, arbitrary units; AOA antioxidant activity, nA per second.
This fact indicates an expressed activation of pro-oxidant component which in turn leads to the depletion of low-molecular antioxidant factors and the development of OS. Less pronounced changes of prooxidant indicators were observed in the animals from group 3, which can be explained by lower toxic load on their non-specific defense system, more rapid neutralization of endogenous toxic substances in the liver, as well as immunomodulatory effects of WMIC with low deuterium content that reduces inflammatory changes in the animals.

All these diverse effects associated with influence of WMIC with low deuterium content to the rats can be explained by a number of mechanisms that are realized at the molecular and cellular levels in vivo. Thus, the consumption of WMIC with low deuterium content leads to the chemical exchange reactions of $\mathrm{H}_{2} \mathrm{O}$ to $\mathrm{D}_{2} \mathrm{O}$ and NDO in cells and to the faster $\mathrm{H} \pm \mathrm{D}$ exchange in hydroxyl, sulfhydryl and amino groups of all organic compounds including proteins, nucleic acids, lipids, sugars that can have an influence on low-molecular component of AOS, which have thiol ($\mathrm{SH})$ and hydroxyl $(-\mathrm{OH})$ groups as one of the main factors. In addition, the presence of deuterium in biological systems leads to changes in the structure and properties of nucleic acids and proteins during the formation of dynamic short-hydrogen (deuterium) bonds, one of the most important for the structure of macromolecules, which can reduce the activity of anti-radical protection enzymes (catalase, superoxide dismutase, glutathione peroxidase, glutathione reductase), thereby reducing the body's antioxidant capacity. In turn, WMIC with low deuterium content restores the enzyme component of AOS while reducing tissue deuterium content both through direct interaction with the $-\mathrm{OH}$ or -SH groups in the active centers and by the activation of transcription due to changes in oscillating moments in the chains of nucleic acid molecules and facilitating their energetic interaction with enzymes providing the genetic code reading.

Also there is violation of ion transport in the cell membrane against the background of high content of deuterium as well as an increase of biomembrane resistance which has particularly negative effect on its excitability [48]. Therefore the use of WMIC with low deuterium content eliminates these negative factors, reduces the viscosity of the membrane, increases its permeability for ions and improves signal transduction of primary and secondary messengers that in turn restores adequate energy exchange in the tissues and reduces prooxidant load at cell structures. In this case, the effects of WMIC will be similar to other indirect antioxidants of our body (e.g., to hormones).

\section{Conclusion}

Thus, it should be noted that blood plasma demonstrates reliable reduction in deuterium concentration when using WMIC with low deuterium content that continues until the values of 90$100 \mathrm{ppm}$ thereafter remaining practically unchanged. At the same time, WMIC with low deuterium content affects prooxidantantioxidant system of the body reducing the intensity of FRRO and restoring the capacity of the endogenous AOS. The largest direct and indirect antioxidant WMIC effect is observed in blood plasma and in hepatocytes, whereas the intensity of free radical processes of the excretory system varies less substantially when giving WMIC to the diet. At the same time the positive effect of WMIC with low deuterium content on inflammatory processes is explained by its possible immunomodulatory effect that reduces the negative effects of bacterial endotoxin substances on the body. All of this allows us to consider WMIC as a promising agent for the 
correction of nutritional imbalance of prooxidant-antioxidant system of the body.

\section{Conflict of interest}

This study was supported by Grant of the Russian Federation President for state support of young Russian scientists (1568.2014.4), the state task of the Ministry of Education and Science of the Russian Federation, the project No. 1269.

\section{References}

1. Dubinina EE. Some features of enzymatic antioxidant defense functioning of human plasma. Biochemistry 1993; 58(2): 268-273. Russian

2. Young IS, Woodside JV. Antioxidants in health and disease. J Clin Pathol 2001; 54: 176-186. (PMID: 11253127) (PMCID: PMC1731363) (doi: 10.1136/jcp.54.3.176)

3. Skulachev VP. Phenoptosis: programmed death of the organism. Biochemistry (Mosc) 1999; 64(12): 1679-1688. (PMID: 10648966)

4. Kulinskiy VI. Active oxygen forms and oxidative modification of macromolecules: use, harm and protection. Soros Educational Journal 1999; (1): 2-8. Russian

5. Vladimirov YuA. Free radicals in biological systems. Soros Educational Journal 2000; (12): 13-19. Russian

6. Boldyrev AA. The role of active oxygen forms in the vital activity of neuron. Advances of Physiological Sciences 2003; 34(3): 21-34.

7. Vladimirov YuA. Active oxygen and nitrogen forms: implications for diagnosis, prophylaxis and therapy. Biochemistry 2004; 69(1): 5-7. Russian

8. Davies KJA. Oxidative stress, antioxidant defenses, and damage removal, repair, and replacement systems. IUBMB Life 2000; 50: 279289. (PMID: 11327322) (doi: 10.1080/713803728)

9. Gomazkov OA. Oxidative stress at molecular, cellular and organ levels. Biochemistry 2003; 68(7): 1005-1006. Russian

10. Stern A. Oxidative stress, cell activation and viral infection. Basel: Birkhauser Verlag, 1994.

11. Balabolkin MI, Klebanova EM. The role of oxidative stress in the pathogenesis of vascular complications of diabetes. Problems of Endocrinology 2000; 46(6): 29-34. Russian

12. Christen Y. Oxidative stress and Alzheimer's disease. Am J Clin Nutr 2000; 71: 621-629.

13. Balabolkin MI, Kreminskaya VM, Klebanova EM. The role of oxidative stress in the pathogenesis of diabetic neuropathy and the possibility of its correction by means of administering $\alpha$-lipoic acid. Problems of Endocrinology 2005; 51(3): 22-33. Russian

14. Shestakova SA, Stepanov RP, Grigorenko GA, Fedorova NV Tishkovskaya YuN. Antioxidant protection and structural changes in the brain of rats with experimental diabetes mellitus. Problems of Endocrinology 2006; 52(5): 37-43. Russian

15. Joshipura KJ, Hu FB, Manson JE, Stampfer MJ, Rimm EB, Speizer FE, et al. The effect of fruit and vegetable intake on risk for coronary heart disease. Ann Intern Med 2001; 134: 1106-1114. (PMID: 11412050) (doi: 10.7326/0003-4819-134-12-200106190-00010)

16. Wu X, Beecher GR, Holden JM, Haytowitz DB, Gebhardt SE, Prior RL. Lipophilic and hydrophilic antioxidant capacities of common foods in the United States. J Agric Food Chem 2004; 52(12): 4026-4037. (PMID: 15186133) (doi: 10.1021/jf049696w)

17. Tutelian VA, Lashneva NV. Biologically active substances of plant origin. Catechins: food sources, bioavailability, the effect on metabolic enzymes of xenobiotics. Nutrition 2009; 78(4): 4-20.

18. Baryshev MG, Dzhimak SS, Basov AA, Bolotin SN, Kashaev DV, Fedosov SR, et al. NMR, EPR, and mass spectroscopy estimates of the antiradical activity of water with modified isotope composition.
Bulletin of the Russian Academy of Sciences: Physics 2012; 76(12): 1349-1352. (doi: 10.3103/S1062873812120039)

19. Bild W, Năstasă V, Haulică I. In vivo and in vitro research on the biological effects of deuterium-depleted water: Influence of deuterium-depleted water on cultured cell growth. Rom J Physiol 2004; 41(1-2): 53-67. (PMID: 15984656)

20. Somlyai G. Naturally occurring deuterium is essential for the normal growth rate of cells. FEBS Letters 1993; 317(1-2): 1-4. (PMID: 8428617) (doi: 10.1016/0014-5793(93)81479-J)

21. Cong FS, Zhang YR, Sheng HC, Ao ZH, Zhang SY, Wang JY. Deuteriumdepleted water inhibits human lung carcinoma cell growth by apoptosis. Exp Ther Med 2010; 1(2): 277-283. (PMCID: PMC3445928)

22. Bild W, Stefanescu I, Haulica I, Lupuşoru C, Titescu G, Iliescu R, Nastasa V. Research concerning the radioprotective and immunostimulating effects of deuterium-depleted water. Rom J Physiol 1999; 36(3-4): 205218. (PMID: 11797936)

23. Rakov DV, Erofeeva LM, Grigorenko DE, et al. Impact of water with low deuterium (heavy stable hydrogen isotope) and oxygen 180 content on the development of radiation damage after gamma irradiation at a low dose-rate. Radiation Biology. Radioecology 2006; 46(4): 475-479. Russian

24. Rakov DV. Effect of water with low deuterium and oxygen 180 content on the development of radiation damage after gamma irradiation. Aerospace and Environmental Medicine 2007; 41(3): 36-39. Russian

25. Kolesova OE, Pomytkin IA. Impact of natural concentration of heavy isotopomers of water on the rate of generation of $\mathrm{H} 2 \mathrm{O} 2$ by mitochondria. Bulletin of Experimental Biology and Medicine 2006; (11): 514-516. Russian

26. Olariu L, Petcu M, Cuna S. The role of deuterium depleted water (ddw) administration in blood deuterium concentration in $\mathrm{Cr}(\mathrm{VI})$ intoxicated rats. Lucrări ştiintifice medicină veterinară 2010; XLIII(2): 193-196.

27. Frolov FYu, Baryshev MG, Bolotin SN, Dzhimak SS. A method for producing of biologically active drinking water with low content of deuterium. Patent [RUS] no.2438765. Priority date 25.05.2010. Publication date 10.01.2012.

28. Frolov FYu, Baryshev MG, Lomakina LV, Dzhimak SS. A method for producing of biologically active drinking water with low content of deuterium. Patent [RUS] no.2438766. Priority date 25.05.2010. Publication date 10.01.2012.

29. Bikov IM, Pavlyuchenko II, Basov AA, Fedosov SR. Comparative antioxidant capacity of some domestic and imported tea drinks. Successes of Contemporary Science 2005; (10): 40. Russian

30. Bahvalov NS, Zhidkov NP, Kobelkov GM. Numerical methods. Russia: Fizmatlit Publ., 2001; 630 p. Russian

31. Borovik ES, Eremenko VV, Milner AS. Lectures on magnetism. Russia: Fizmatlit Publ., 2005; 512 p. Russian

32. Azhipa Yal. Medical and biological aspects of electron paramagnetic resonance method. USSR: Nauka, 1983; 528 p. Russian

33. Pulatova MK, Rihireva GT, Kuropteva ZV. Electron paramagnetic resonance in molecular radiobiology. USSR: Energoatomizdat, 1989; $232 \mathrm{p}$

34. Vladimirov YuA, Proskurina EV, Izmaylov DY. Kinetic chemiluminescence as a method of studying of free radicals reactions. Biophysics 2011; 56(6): 1055-1062.(doi: 10.1134/S0006350911060200)

35. Basov AA, Pavlyuchenko II, Plaxin AM, Fedosov SR. Using the analogto-digital converter in the system for collecting and processing data from hemi lumen tester LT-1. Bulletin of New Medical Technologies 2003; 10(4): 67-68. Russian

36. Basov AA, Bikov IM. Comparative characteristics of the antioxidant capacity and the energy value of some foods. Vopr Pitan 2013; 82(3) 77-80. (PMID: 24006756)

37. Pavlyuchenko II, Basov AA, Bikov IM, Orlova SV. Integral methods for assessing the level of endogenous intoxication and peroxidation of biomolecules in acute and chronic diseases. Allergology and Immunology 2004; 5(4): 551-554. Russian 
38. Yashin YaA. A flow-injection system with amperometric detection for selective determination of antioxidants in foodstuffs and drinks. Russian Journal of General Chemistry 2008; 78(12): 2566-2571. (doi: 10.1134/S1070363208120360)

39. Basov AA, Baryshev MG, Dzhimak SS, Bykov IM, Sepiashvili RI, Pavliuchenko II. The effect of consumption of water with modified isotope content on the parameters of free radical oxidation in vivo. Fiziol Zh 2013; 59(6): 49-56. Russian (PMID: 24605591)

40. Basov AA, Baryshev MG, Dzhimak SS, et.al. Effect of water with a modified isotopic composition on the performance on the intensity of free-radical processes in experiment of laboratory animals. Allergology and Immunology 2012; 13(4): 314-320. Russian

41. Baryshev MG, Dzhimak SS, Bolotin SN, Frolov VYu, Dolgov MA. Technologies for obtaining water with low content of deuterium. International Journal of Engineering Research and Applications 2013; 3(1): 523-526.

42. Barishev MG, Frolov VU, Dzhimak SS. The methods of production of deuterium depleted water. Ecological Bulletin of Research Centers BSEC 2013; (1): 13-17. Russian

43. Basov AA, Baryshev MG, Dzhimak SS, et al. NMR and EPR study of deuterium depleted water effect on the laborotary animals' prooksidant-antioksidant system. Ecological bulletin of research centers BSEC 2011; (3): 16-20. Russian

44. Baryshev MG, Dzhimak SS, Kas'yanov GI, et al. Application of water with the modified isotope structure and $\mathrm{pH}$ in the meat industry. Izvestiya vuzov. Food technology 2012; (2-3): 42-44. Russian

45. Baryshev MG, Bolotin SN, Dzhimak SS, et al. Electrochemical method of production and biological properties of deuterium depleted water. Science of Kuban 2010; (3): 18-21.

46. Mirkhaydarov AR. Chemiluminescence study of blood and urine in patients with critical conditions. In: Abstracts of scientific and practical conf. with international participation "Free radicals, antioxidants and human diseases", 2001: 212-214. Russian

47. Litvinova MG, Basov AA, Bikov IM. Indicators of free radical oxidation in the blood and oral fluid of patients with ischemic heart disease and diabetes type 2. Kuban Research Medical Vestnik 2012; (3): 94-98. Russian

48. Lobyshev VN, Kalinichenko LP. Isotope effects of $D_{2} \mathrm{O}$ in biological systems. Nauka 1978: 215. Russian

Authors:

Oxana Artsybasheva - PhD student, Radiophysics chair, Kuban State University, Krasnodar, Russia;

Yekaterina Barysheva - PhD student, Pathophysiology chair, Kuban State Medical University, Krasnodar, Russia;

Denis Shashkov - PhD student, Radiophysics chair, Kuban State University, Krasnodar, Russia;

Roman Vlasov - PhD student, Radiophysics chair, Kuban State University, Krasnodar, Russia;

Elena Tekutskaya - PhD, docent of radiophysics chair, Kuban State University, Krasnodar, Russia. 